\title{
Comparison of gabapentin and oxycodone- acetaminophen used for pre-emptive analgesia in patients undergoing double-port thoracoscopic pulmonary surgery: a randomized, double-blind, controlled trial.
}

Li Li ( $\nabla$ lili5917@hotmail.com )

Centrl South University Xiangya Second Hospital https://orcid.org/0000-0003-4061-8078

XiJuan Li

Central South University Xiangya Second Hospital

JinMei Shen

Central South University Xiangya Second Hospital

LiJun Zhou

Central South University Xiangya Second Hospital

Research article

Keywords: gabapentin; oxycodone-acetaminophen; pre-emptive analgesia;thoracoscopic pulmonary surgery

Posted Date: November 7th, 2019

DOI: https://doi.org/10.21203/rs.2.16815/v1

License: (c) (1) This work is licensed under a Creative Commons Attribution 4.0 International License. Read Full License 


\section{Abstract}

Background This study was designed to determine whether gabapentin is not inferior to the oxycodoneacetaminophen group used as pre-emptive analgesia in reducing post-operative pain. The post-operative pain of patients undergoing thoracoscopic pulmonary surgery, a routine operation procedure, is also a clinical problem that urgently needs to be further explored. We hypothesized that gabapentin is not inferior to oxycodone-acetaminophen.Methods Ninety patients were randomly divided into group $A, n=30$; group $B, n=30$; and group $C, n=30$. Patients in group A received oral gabapentin ( $300 \mathrm{mg}) 2 \mathrm{~h}$ before surgery, similarly patients in group B received oral oxycodone-acetaminophen (330mg);Group C did not take any oral drugs; all patients were given self-controlled intravenous analgesia after surgery. NRS scores post-operative, opioid consumption 48 hours post-operative, analgesic-related adverse events, post-operative chronic pain after 2 months, were recorded.Results The NRS scores and opioid consumption 48 hours post-operative of intervention groups were significantly lower than the control group, and did not increase analgesic-related adverse events. The incidence of chronic pain 2 months post-operative in groups $A$ and $B$ was significantly lower than group C.Conclusion Oral gabapentin and oxycodone-acetaminophen alleviated the pain post-operative, reduced opioid consumption postoperative,promoted the recovery.

\section{Background}

Thoracoscopic pulmonary surgery due to advantages, such as less traumatic compared with open surgery, has become a more mature surgical approach compared with traditional open pulmonary surgery in comprehensive hospitals; however, although double-port thoracoscopic pulmonary surgery is less traumatic, the post-operative pain is still severe due to tissues and intercostal nerve injury. Intense pain stimulation during the peri-operative period not only triggers various adverse stress reactions in the body, such as metabolic disorders, immunosuppression, and hemodynamic abnormalities[1], increasing the incidence of peri-operative complications. Post-operative pain will limit deep breathing and effective coughing, which antagonizes the recovery of lung function and will increase the incidence of pulmonary complications, thus extending the length of the ICU stay. Post-operative activity limitations also increase thrombogenesis[2], and post-operative pain also increase the psychological burden of patients. Previous studies have disclosed that unalleviated post-operative pain was associated with various complications such as deep veins thrombosis, increased heart rate, and blood pressure[3][4]. Thus, a better perioperative analgesic protocol should relieve patients from post-operative pain, reduce the incidence of post-operative complications, and promote post-operative recovery. At present, the prevalent postoperative analgesia protocol primarily consists of opioid-based post-operative continuous intravenous analgesia; however, overuse of opioid-based post-operative continuous intravenous analgesia may lead to post-operative adverse events, which is not conducive to rapid post-operative recovery and is not in accordance with the principle of enhanced recovery after surgery(EARS). Therefore, we sought to develop a better analgesic protocol. Pre-emptive analgesia is not a concept refers to a treatment that starts before surgery and is intended to prevent central sensitization caused by surgical incisional injury and other 
inflammatory responses to surgery[5][6]proposed by international scholars in the early 20th century. There are a large number of clinical drugs used for pre-emptive analgesia, mainly including local anesthetics, opioids, non-steroidal anti-inflammatory drugs, N-methyl-D-aspartate(NMDA) receptor antagonists, and alpha 2-adrenergic receptor agonists. Gabapentin is a structural analog of gammaaminobutyric acid, which are widely distributed in the central and peripheral nervous systems, inhibits calcium influx, and reduces the release of excitatory neurotransmitters in pain pathways. In recent years, scientific research has confirmed that gabapentin, antiepileptic drug, has a good pre-emptive analgesic effect during spinal surgical procedures and lower abdominal surgery[7][8].Oxycodone-acetaminophen is a prescription tablet of oxycodone hydrochloride and acetaminophen. According to reports, oxycodone can prevent the conduction of harmful stimulation induced by central nervous impulses. Simultaneously, before the harmful stimulus reaches the central nervous system, the excitability of the central nervous system is inhibited and the sensitization of the central nervous system is suppressed, thus achieving a good analgesic effect. Acetaminophen is a non-steroidal anti-inflammatory and analgesic drug that has an inhibitory effect on the activity of Cox-2. There are no case-control studies involving the pre-emptive analgesia of gabapentin and oxycodone-acetaminophen in patients undergoing double-hole thoracoscopic pulmonary surgery. After fully searching and reviewing the relevant literature combined with clinical observations, this study selected a clinical case-control study to compare gabapentin or oxycodone-acetaminophen combined with post-operative self-controlled continuous intravenous analgesia and single post-operative self-controlled continuous intravenous analgesia. A preliminary evaluation of the effects of gabapentin and oxycodone-acetaminophen for pre-emptive analgesia in such patients was performed. The essence of this study is the comparison of the effects of non-opioid and opioid used as pre-emptive analgesia.Therefore, a preliminary clinical basis for the use of advanced analgesia in patients undergoing double-port thoracoscopic pulmonary surgery is provided.We hypothesized that gabapentin is not inferior to oxycodone.

\section{Methods}

\section{Patients}

This randomized controlled trial enrolled patients from the Second Xiangya Hospital of Central South University from 1 May 2019 to 30 June 2019 who underwent double-port thoracoscopic pulmonary surgery for lung disease. Patients who met the criteria(vide infra) and signed the written informed consent were randomly divided into gabapentin (group A, 30 cases), oxycodone-acetaminophen(group B, 30 cases) and the control group (group C, 30 cases) using Excel 2010 (Microsoft Inc. Redmond, WA, USA) to generate random numbers. Because of the invasive nature of the interventions, neither the trial participants nor the investigators were masked to group allocation. The administration of pre-emptive analgesics was performed by nurses on the ward, and intra-operative anesthesia management and postoperative follow-up were performed by two anesthesiologists respectively. Mutual assistance was not allowed. This study was approved by the Ethics Committee of Second Xiangya Hospital of Central South University and was registered in China clinical trial registry. 
The inclusion criteria were as follows: (1) patients with pulmonary lesions confirmed by imaging examination; (2) surgery which could be completed by double-port thoracoscopy based on a perioperative evaluation; (3) patients $\geq 18$ years and $\leq 70$ years of age.

The exclusion criteria were as follows: (1)patients who simultaneously were participating in other clinical research; (2)patients with organs(such as heart, lung, liver, and kidney) with serious dysfunction; (3)patients who are allergic to gabapentin and oxycodone-acetaminophen or similar drugs; (4)patients with language communication barriers; (5)patients with a history of drug abuse; (6)patients with a mental illness; (7)patients who cannot understand the NRS; (8)patients who were born with no pain sensation; (9)patients with a history of chronic pain; (10)patients in poor general health; (11)patients with asthma or other serious respiratory diseases; (12)patients in whom the operative method was changed; (13)patients with peri-operative massive blood loss or shock; (14)patients who died during observation period; and (15)patients who failed to complete evaluation because of severe post-operative complications.

\section{Procedures}

Two hours before surgery, the nurses on the ward administered the pre-emptive analgesic drugs in accordance with the established pre-emptive analgesic scheme. Patients in group A received oral gabapentin (300 mg) $2 \mathrm{~h}$ before surgery, similarly patients in group B received oral oxycodoneacetaminophen $(330 \mathrm{mg})$;Group $\mathrm{C}$ did not take any oral drugs; all patients were given self-controlled intravenous analgesia after surgery. After entering the operating room, peripheral venous channel was routinely established. Vital signs and EEG bispectral index (BIS) was monitored.All patients in the three groups received same intravenous induction cocktail(midazolam $[0.06 \mathrm{mg} / \mathrm{kg}]$, sufentanil $[0.5 \mathrm{ug} / \mathrm{kg}]$, vecuronium $[0.1 \mathrm{mg} / \mathrm{kg}]$, and etomidate $[0.2 \mathrm{mg} / \mathrm{kg}])$, cis-atracurium $(0.1 \mathrm{mg} / \mathrm{kg} / \mathrm{h})$ to maintain intraoperative muscle relaxation, and the depth of anesthesia was maintained by remifentanil and propofol. The BIS was maintained between 40 and 60 during the surgery. Sufentanil can be appropriately added during the operation according to the depth of anesthesia. After the end of the operation, the doublebronchial catheter should be replaced with a single-lumen endotracheal catheter, therefore the continuous injection of remifentanil, propofol and cis-atracurium was not be stopped until the single-lumen endotracheal catheter replacement was completed. The post-operative patient self-controlled intravenous analgesia pump was configured in the same manner. Sufentanil $(1.5 \mathrm{ug} / \mathrm{kg})$ and ondansejoon $(16 \mathrm{mg})$ diluted to a final volume of $100 \mathrm{ml}$ in normal saline. The parameters of the analgesic pump included a 2 $\mathrm{ml} \cdot \mathrm{h}^{-1}$ continuous infusion volume $\left(0.03 \mu \mathrm{g} \cdot \mathrm{kg}^{-1} \cdot \mathrm{h}^{-1}\right)$, a $2 \mathrm{ml}$ bolus, a $10 \mathrm{~min}$ lockout time, and an 8 $\mathrm{ml} \cdot \mathrm{h}^{-1}$ maximum in-fusion volume. If the post-operative pain score at rest was $\geq 4$, the patient was given self-controlled analgesia firstly, and if there was no relief $10 \mathrm{~min}$ after 1 bolus dose, the patient was given supplementary analgesics. The mean arterial pressure (MAP) and heart rate (HR) were recorded upon entry to the operating room(T0), at the time of the skin resection (T1), at the time of the thoracoscopy was placed in the chest(T2), $1 \mathrm{~h}$ post-operatively(T3), $2 \mathrm{~h}$ post-operatively(T4), 4h post-operatively(T5), 8h post-operatively(T6), 12h post-operatively(T7), 16h post-operatively(T8). The length of the operation, length of anesthesia, and extubation time were recorded. In addition, we recorded the primary endpoint including the NRS scores at rest and while coughing at 4, 8, 12, 24, 48h post-operative, intra-operative 
propofol and remifentanil consumption, opioid consumption post-operative; the secondary endpoint including the amount of time out of bed on the first post-operative day, the awaken times due to pain during the frist night post-operative, the frequency of patient self- controlled analgesia within $48 \mathrm{~h}$, first time to remedy analgesia post-operative, and the length of the ICU stay post-operative, the length of the hospital stay post-operative, and the incidence of analgesic-related adverse events such as post-operative agitation, fatigue, nausea and vomiting, dizziness, respiratory depression ( $R R<10$ and pulse oxygen saturation $<94 \%$ on oxygen inhalation), the incidence of post-operative chronic pain 2 months postoperative were recorded.

\section{Statistical analysis}

All statistical analyses of the data were performed using IBM SPSS Statistics V21.0. The sample size in each group was 30 patients, and an $\alpha=0.05$ (statistically significant level) and a $1-\beta=0.8$ test power were set. One-way analysis of variance was used for comparison between groups, and one-way repeated measures analysis of variance was used for comparison within groups. The chi-square test was used to compare the count data between groups. A P $<0.05$ was considered to be statistically significant.

\section{Results}

Ninety patients were included in this study, including group A $(n=30)$, group $B(n=30)$ and group $C(n=30)$. Two patients in group $A$ received general anesthesia combined with a nerve block, 1 patient in group $B$ received frequent post-operative ventricular premature loss, and 1 patient in group $C$ was converted to an open thoracotomy. Twenty-eight, twenty-nine, twenty-nine patients respectively in group A, B, and C completed the study. The comprehensive standard of the experimental report is shown in Figure 1.

There were no statistically significant differences in gender composition, age, ASA classification, body mass index (BMI), loss of follow-up rate, hypertension, and diabetes among the three groups $(P>0.05)$, as shown in Table 1.

There was no significant difference in the NRS scores of the three groups at $4 \mathrm{~h}$ at rest $(P>0.05)$, the NRS scores of groups $A$ and $B$ at $4 \mathrm{~h}$ when coughing were significantly lower than group $C(P<0.05)$, while there was no significant difference between groups $A$ and $B(P>0.05)$; the NRS scores at $8 h, 12 h, 24 h$ and $48 h$ at rest and while coughing were significantly lower in groups $A$ and $B$ than group $C(P<0.05)$; there was no significant difference between groups $A$ and $B(P>0.05)$, as shown in Figure 2 and Table 2; intra-operative propofol consumption in groups $A$ and $B$ was significantly lower than group $C(P<0.05)$; intra-operative propofol consumption in group $B$ was lower than group $A(P<0.05)$; the intra-operative consumption of remifentanil in groups $A$ and $B$ was significantly lower than group $C(P<0.05)$; there was no significant difference between groups $A$ and $B(P>0.05)$, as shown in Table 3; The opioid consumption post-operative in the intervention groups was significantly lower than the control group $(P<0.05)$,as shown in Table 4.

There was no significant difference in the peri-operative HR and MAP between the three groups $(P>0.05)$, as shown in Table 5 and Table 6; there was no significant difference in the operative period, 
anesthesia period, and extubation time $(P>0.05)$, as shown in Table 7; there was no significant difference in the length of hospital stay $(P>0.05)$ between in the three groups, while the post-operative length of the ICU stay in groups $A$ and $B$ was significantly shorter than group $C(P<0.05)$, as shown in Table 8 ; the awaken times due to pain on the frist post-operative night in groups $A$ and $B$ were significantly less than group $C(P<0.05)$; there was no significant difference between groups $A$ and $B(P>0.05)$; the amount of time out of bed on the first post-operative day in groups $A$ and $B$ were significantly longer than group $C$ $(P<0.05)$, the amount of time out of bed on the first post-operative day in group $B$ was significantly longer than group $A(P<0.05)$; the frequency of self-controlled analgesia within $48 \mathrm{~h}$ after surgery in groups $A$ and $B$ were significantly less than group $C(P<0.05)$,there was no significant difference between groups $A$ and $B(P>0.05)$; there was no significant difference of frist time to remedy analgesia post-operative between the groups $A, B$ and $C(P>0.05)$, as shown in Figure 3 and Table 4,there was no significant difference in the incidence of post-operative dysphoria, respiratory depression, dizziness, weakness, and nausea or vomiting in the three groups $(P>0.05)$; the incidence of chronic pain in groups $A$ and $B 2$ months postoperative was significantly lower than group $C(P<0.05)$, there was no significant difference between

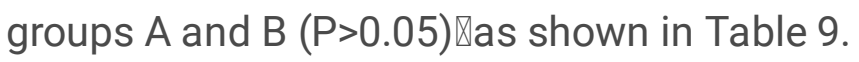

\section{Discussion}

Thoracic surgical incisions are characterized by traumatic injury to the intercostal nerves and tissues. The resulting nerve damage is often irreversible and is an important cause of post-operative acute and chronic pain. Pre-emptive analgesia methods have been used alone or in combination for peri-operative analgesia. The meta-analyses performed by Cliffk-s [9] and Ong et al[10] showed that pre-emptive analgesia interventions reduce post-operative pain scores and post-operative remedial analgesia. Indeed, some studies have demonstrated the benefits of gabapentin during the peri-operative period and confirmed that gabapentin has a good analgesic effect in spinal surgical procedures and lower abdominal surgery [5]. Similarly, gabapentin has been shown to reduce pain scores and analgesics as an adjuvant analgesic drug during oral surgery [11]. Furthermore, oxycodone-acetaminophen has been shown to have a good analgesic effect after oral surgery [12]. The meta-analysis conducted by Jiaqi et al[13] concluded that taking a single dose of pregabalin or gabapentin before surgery has a significant effect on reducing opioid consumption and post-operative pain. The meta-analysis conducted by Liu et al[14] showed that oral administration of oxycodone-acetaminophen pre-operatively may have a good analgesic effect in plastic and cosmetic surgeries. Young [15] reported significant efficacy in oral pain after surgery with butorphanol/acetaminophen and oxycodone/acetaminophen.In this study we also showed that gabapentin and oxycodone-acetaminophen, when used as pre-emptive analgesia in patients undergoing double-hole thoracoscopic pulmonary surgery, were safe and effective.

Alayed et al examined the evidences in various databases and concluded that preoperative gabapentin not only decreased the visual analogue score but also reduced the incidence of nausea and vomiting[16]. Tomar GS [17] evaluated the analgesic effects of three different doses $(400 \mathrm{mg}, 800 \mathrm{mg}, 1200 \mathrm{mg})$ of oral gabapentin after inguinal hernia under spinal anesthesia, and found that gabapentin from $400 \mathrm{mg}$ to $1200 \mathrm{mg}$, with increasing dose, the side effects are even more serious but the analgesic effect does not 
increase. Pandey et al[18],in their study between gabapentin $(300 \mathrm{mg})$ and tramadol $(100 \mathrm{mg})$ in patients undergoing laparoscopic cholecystectomy, concluded that the pre-emptive use of oral gabapentin significantly decreases post-operative pain and requirement of rescue analgesia in laparoscopic cholecystectomy. A study conducted by Kochhar A[19] found that a single pre-dose of pregabalin (150 $\mathrm{mg}$ ) or gabapentin $(300 \mathrm{mg}$ ) was equally effective in relieving pain as part of multimodal treatment after laparoscopic cholecystectomy and there are no side effects. A study conducted by Kang HS[20] found that the most suitable dose of gabapentin in reducing the optimal dose of fentanyl after gynecological surgery was $300 \mathrm{mg}$. In addition, the bioavailability of gabapentin decreases with increasing dose; therefore, we chose gabapentin(300mg) as the experimental dose. Kogan A1 et al[21] found that oxycodone-acetaminophen (5mg / 325mg) has better analgesic effect and less side effects than sustained-release oxycodone $(10 \mathrm{mg})$ in oral surgery EARS anesthesia.Davis KM et al[22] found that oral analgesia with oxycodone-acetaminophen may offer superior pain after cesarean delivery with fewer side-effects as compared with morphine patient-controlled analgesia.A meta-analysis [23] showed that high doses of oxycodone-acetaminophen were associated with an increase in analgesic-related adverse events, but these adverse events were often described as mild or moderate and were not associated with withdrawal. Therefore, we chose oxycodone-acetaminophen $(330 \mathrm{mg})$ containing oxycodone $(5 \mathrm{mg})$ and acetaminophen(325mg).

Data from the current study indicated that using oral gabapentin and oxycodone-acetaminophen as preemptive analgesia did not cause significant hemodynamic fluctuations whether pre-, intra- or postoperative. Therefore, the use of gabapentin or oxycodone-acetaminophen for pre-emptive analgesia did not delay recovery after general anesthesia in the absence of statistical differences between operative time and anesthesia time. From this study we may speculate that the use of pre-emptive analgesia can indirectly reduce the incidence of pulmonary complications in the intervention patients due to the decrease of length of ICU stay. Of note, there were no statistical differences in the length of hospital stay between the three groups of patients. We speculate that part of the reason may be that the patients are not discharged from the hospital on weekends. It has been shown that pre-administration of gabapentin reduced the consumption of opioids within $24 \mathrm{~h}$ after surgery [24]. It has also been shown that ibuprofen, when combined with oxycodone-acetaminophen, is effective in treating chronic pain and reducing the consumption of narcotic analgesics [25]. This finding is consistent with our findings, which indicated that pre-operative oral gabapentin $(300 \mathrm{mg})$ or oxycodone-acetaminophen $(330 \mathrm{mg})$ reduced the consumption of intra-operative propofol, remifentanil and opioids consumption post-operative. The results are also consistent with the findings reported by Steinberg [26], suggesting that the use of gabapentin reduces the required dose of general anesthetic drugs. Moreover, the results coincide with the findings reported by Alayed et al[16]. A number of studies have confirmed that pre-emptive analgesia can effectively reduce post-operative pain scores [9][10][13]. Similarly, the results from this study, suggested that the intervention groups received more adequate analgesia in the early post-operative period than the control group, and there was no statistical difference in pain scores between gabapentin(300mg) and oxycodoneacetaminophen[(oxycodone 5mg) and (acetaminophen 325)]. This finding can be explained by that administration of small doses of analgesics before pain stimulation, thus blocking the nociceptive effect, 
enhancing post-operative analgesia, and relieving pain. Early ambulation is a crucial link for the postoperative recovery and can increase a patient's vital capacity and decrease lung complications for patients undergoing thoracoscopic lung surgery. Of note, our results revealed that oxycodoneacetaminophen is more effective than gabapentin when used as pre-emptive analgesia,especially postoperative analgesia with activities.. Pain has been shown to be associated with poor sleep quality, awakenings, and a shorter sleep time [27]. The relationship between pain and sleep disorders has been verified in some studies and the evidence of relationships of pain with sleep quality, reduced sleep time, lower sleep efficiency, and more awakenings has been determined[28][29]. Several nights of poor sleep has been shown to decrease pain thresholds[30]. Gabapentin(300mg) or oxycodoneacetaminophen[(oxycodone $5 \mathrm{mg}$ ) and (acetaminophen 325)], when used as pre-emptive analgesia combined with post-operative intravenous analgesia,can improve sleep quality equally early after surgery. Farzi [31] reported that gabapentin or tramadol can effectively reduce post-operative pain without any side effects. For severe pain, the early oral administration of analgesia is safe and effective. Compared with the tramadol group, the incidence of adverse reactions was lower in the oxycodone-acetaminpohen group [32]. It has also been confirmed that peri-operative oral gabapentin effectively reduces postoperative pain scores, anesthetic consumption, and nausea and vomiting [16]. A meta-analysis conducted by Grant [33] (44 studies, $n=3489$ ) also revealed that gabapentin is associated with a reduction in post-operative nausea and vomiting. Richards et al[34][35] showed that oxycodoneacetaminophen, when used in orthopedic patients, has the characteristics of an analgesic effect and few adverse reactions. Tiippana [36] conducted a meta-analysis involving 22 consecutive randomized clinical comparative studies on the application of gabapentin and pregabalin during the peri-operative period to evaluate the effect of pre-operative oral gabapentin on post-operative analgesia, which revealed that gabapentin effectively reduces post-operative pain, opioid consumption, and opioid-related adverse events after surgery. This is consistent with our results $₫$ which the morphine consumption in the intervention group was statistically less than the control group. However, no satistically significant difference in the incidence of nausea and vomiting, including agitation, fatigue, and dizziness, existed between the intervention and control groups in the current study, which may be explained by the dose of gabapentin(300mg) we selected did not reduce the incidence of nausea and vomiting. At the same time, we concluded that oxycodone-acetaminophen did not increase post-operative analgesia-related adverse events in the context of enhanced peri-operative analgesia. Therefore, it can be seen from this study that gabapentin (300 mg) and oxycodone-acetaminophen[(oxycodone 5mg) and (acetaminophen 325mg)] decrease the post-operative pain scores equally in patients undergoing double-port thoracoscopic pulmonary surgery without increasing post-operative adverse-related reactions.

ERAS has become a popular management mode for contemporary surgical procedures that aims to reduce peri-operative complications, accelerate post-operative recovery, and shorten the hospital stay [37]. For patients undergoing thoracic surgery, a better peri-operative analgesic protocol is to relieve patients from post-operative pain, reduce the incidence of post-operative complications, promote the early activities of patients, reduce post-operative atelectasis, decrease post-operative pulmonary infections, and promote recovery. Although it has been clearly confirmed in this study that pre-operative oral 
gabapentin and oxycodone-acetaminophen, when used as pre-emptive analgesia and combined with post-operative self-controlled continuous intravenous analgesia in patients undergoing double-port thoracoscopic lung surgery, are able to provide a better peri-operative analgesia method; however, this study had several shortcomings. Referring to the previous literature, experimental groups recorded the chronic pain rate 4 months, 6 months, or even longer after surgery, but the chronic pain rate was only recorded 2 months after surgery in the current study due to time constraints. The time to remove the tracheal tube was generally long in all three groups. There may be three reasons. First, the anesthetist was asked to replace the double lumen bronchial catheter with a single lumen endotracheal tube after completion of thoracic surgery, which is more convenient for airway management post-operatively. Therefore, infusion of cis-atracurium, propofol, and remifentanil continues until the single-lumen endotracheal tube replacement has been done. Second, because of different indications for extubation between thoracic surgeons and anesthesiologists, the indications for extubation in the thoracic care unit were overly cautious. Due to the shortage of researchers, no professional anesthesiologist was deployed to the thoracic care unit to oversee the extubation. Third, the chest surgery intensive care unit is understaffed and there is a shortage of staff in the intensive care unit, thus tracheal tube extubation was delayed. When we are doing clinical research on clinical analgesia, we should fully consider the individual differences of patients, such as age and gender; however, the complexity of the operation, the length of the operation, and the extent of the surgery cannot be ignored and will inevitably affect the analgesic effect. Our medical workers have to consider some scientific methods to minimize the impact of these factors. In China, the visual analog scale method is widely used to evaluate pain. This method marks the corresponding position of pain by the patient. The physician evaluates pain according to the position marked by the patient. This method is subjective and the test result partially affects the psychological make-up of the patient.

\section{Conclusions}

In summary, pre-emptive analgesia with gabapentin(300mg) and oxycodone-acetaminophen[(oxycodone $5 \mathrm{mg}$ ) and (acetaminophen 325)] equally reduced the opioids consumption post-operative, decreased post-operative NRS scores, improved recovery, reduced the incidence of chronic pain 2 months after surgery, thus improved the quality of prolonged life for the patients. So pre-emptive analgesia of gabapentin(300mg) and oxycodone-acetainophen[(oxycodone $5 \mathrm{mg}$ ) and (acetaminophen 325)] combined with post-operative self-controlled continuous intravenous analgesia is a safe and effective analgesic method.

\section{Abbreviations}


ASA: America Society of Anesthesiologist; BMI: Body Mass Index; HR: Heart Rate; MAP: Mean Arterial Pressure; NRS: Numerical Rating Scale

\section{Declarations}

\section{Ethics approval and consent to participate}

The research protocol was approved by the Medical Ethics Committee of the Second Xiangya Hospital of Central South University, Changsha, China(Number:2019-104), and all the patients signed the written informed consent voluntarily.And this study adheres to CONSORT guidelines.

\section{Consent for publication}

Not applicable.

\section{Availability of data and materials}

The datasets used and/or analyzed during the current study are available from the corresponding author on reasonable request.

\section{Competing interests}

The authors declare that they have no competing interests.

\section{Funding}

This study was funded by Nature Science foundation Commission of Hunan Province (2015JJ4098 to Jinmei Shen). The funding had no role in study design,data collection and analysis,decision to publish,or preparation of the manuscript.

\section{Authors' contributions}

This research was accomplished by three co-authors. The contribution of each author are as follows: XJL was involved in designing the study, accomplishing the work of postoperative follow-up, collecting the most of the data, and drafting the manuscript and instructed the design of the research proto-col; LL participated in designing the intraoperative part of the research protocol and performed the general 
anesthesia processes and participated in designing the recruit-ment criteria and patient recruitment processes; JMS was involved in designing the postoperative part of the research protocol and assisted XJL and LJZ to finish postoperative follow-up.All authors have read and approved the manuscript.

\section{Acknowledgements}

We appreciate the efforts of all health-care providers and the patients and family for their participation in this study. We also acknowledge The Depart-ment of Anesthesiology team and The Department of Thoracic surgery team in Second Xiangya hospital of Central South University for supporting the research.

\section{Author details}

${ }^{1}$ Department of Anesthesiology, The Second Xiangya Hospital of Central South University, 139 Renmin Middle Road, Hunan, China.

\section{References}

[1] Steinthorsdottir KJ『Wildgaard L囚Hansen HJ囚et al.Regional analgesia for video-assisted thoracic

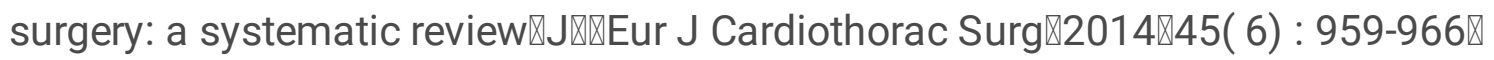

[2] FAN Xiaobing $\mathbb{F}$ AN Xiaobing.Patient 's Analgesic Methods after Thoracoscopic Surgery and Its Research Progress[J].Chinese Continuing Medical Education,2018,10(09):53-55.

[3] Kohli M, Murali T, Gupta R, Khan P, Bogra J. Optimization of Subarachanoid Block by Oral Pregabalin for Hysterectomy. J Anaesth Clin Pharmacol. 2011;27(1):101-105.

[4] Dahl JB, Mathiesen O, Møiniche S. Protective premedication': an option with gabapentin and related drugs? Acta Anaesthesiol Scand. 2010;48(9):1130-1136.

[5]. Lee BH囚Park JO凶Suk KS囚et al.Pre-emptive and multi-modal perioperative pain management may improve quality of life in patients undergoing spinal surgery.Pain Physician. 2013 May-Jun;16(3):E21726.

[6]Savitha KS $₫$ Dhanpal R $₫$ Kothari AN.The Effect of Multimodal Analgesia on Intraoperative Morphine Requirement in Lumbar Spine Surgeries.Anesth Essays Res. 2017 Apr-Jun;11(2):397-400. doi: 10.4103/0259-1162.194553.

[7]Han C, Kuang MJ, Ma JX1, Ma XL.The Efficacy of Preoperative Gabapentin in Spinal Surgery: A MetaAnalysis of Randomized Controlled Trials.Pain Physician. 2017 Nov;20(7):649-661. 
[8]Parikh HG, Dash SK, Upasani CB.Study of the effect of oral gabapentin used as preemptive analgesia to attenuate post-operative pain in patients undergoing abdominal surgery under general anesthesia.Saudi J Anaesth. 2010 Sep;4(3):137-41. doi: 10.4103/1658-354X.71409.

[9]Kelly DJ, Ahmad M, Brull SJ. Preemptive analgesia II: Recent advances and current trends. Can J Anaesth. 2001;48:1091-101.

[10]Cliff K-S Ong, Philipp L, Robin A, Seymour, Brian J. Jenkins The Efficacy of Preemptive Analgesia for Acute Postoperative Pain Management: A Meta-Analysis Anesth Analg. 2005;100:757-73.

[11]Coderre TJ, Kumar N, Lefebvre CD, Yu JSC. Evidence that gabapentin reduces neuropathic pain by inhibiting the spinal release of glutamate. J Neurochem. 2005;94:1131-9.

[12]Chang DJ, Desjardins PJ, Bird SR, Black P, Chen E, Petruschke RA, Geba GP.Comparison of rofecoxib and a multidose oxycodone/ acetaminophen regimen for the treatment of acute pain following oral surgery: a randomized controlled trial.Curr Med Res Opin. 2004 Jun;20(6):939-49.

[13][Jiaqi Hu, Dongdong Huang,Minpu Li, Chao Wu, and Juan Zhang.Effects of a single dose of preoperative pregabalin and gabapentin for acute postoperative pain: a network meta-analysis of randomized controlled trials.J Pain Res. 2018; 11: 2633-2643.]

[14]Liu ZF, Wang XJ, Wang XC, Zhu L, Qiao Q.Clinical study of preoperative analgesia for liposuction.Zhonghua Zheng Xing Wai Ke Za Zhi. 2007 Mar;23(2):128-9.

[15]Young RE.A comparison of analgesic effectiveness of oral butorphanol/acetaminophen, oxycodone/acetaminophen and placebo in hospitalized postsurgical patients.J Med. 1979;10(6):417-28.

[16]Alayed N, Alghanaim N, Tan X, Tulandi T.Preemptive use of gabapentin in abdominal hysterectomy: a systematic review and meta-analysis.Obstet Gynecol.2014 Jun;123(6):12219.doi:10.1097/AOG.0000000000000289.

[17]Tomar GS, Singh F, Cherian G.Role of Preemptive Gabapentin on Postoperative Analgesia After Infraumbilical Surgeries Under Subarachnoid Block-A Randomized, Placebo-Controlled, Double-Blind Study.Am J Ther. 2019 May/Jun;26(3):e350-e357. doi: 10.1097/MJT.0000000000000700.

[18]Pandey CK, Priye S, Singh S, Singh U, Singh RB, Singh PK. Preemptive use of gabapentin significantly decreases postoperative pain and rescue analgesic requirements in laparoscopic cholecystectomy. Can $\mathrm{J}$ Anaesth. 2004;51:358-63.

[19]Kochhar A1, Chouhan K1, Panjiar P1, Vajifdar H1.Gabapentinoids as a Part of Multi-modal Drug Regime for Pain Relief following Laproscopic Cholecystectomy: A Randomized Study.Anesth Essays Res. 2017 Jul-Sep;11(3):676-680. doi: 10.4103/0259-1162.204208. 
[20]Kang HS1, Park HJ1, Choi J1, Park SJ1, Lee SK1. The optimal preemptive dose of gabapentin following gynecologic surgery.Kang HS1, Park HJ1, Choi J1, Park SJ1, Lee SK1.Korean J Anesthesiol. 2009 Mar;56(3):309-312. doi: 10.4097/kjae.2009.56.3.309.

[21]]Kogan A1, Medalion B, Raanani E, Sharoni E, Stamler A, Pak N, Vidne BA, Eidelman LA.Early oral analgesia after fast-track cardiac anesthesia.Can J Anaesth. 2007 Apr;54(4):254-61.

[22]Davis KM1, Esposito MA, Meyer BA.Oral analgesia compared with intravenous patient-controlled analgesia for pain after cesarean delivery: a randomized controlled trial.Am J Obstet Gynecol. 2006 Apr;194(4):967-71.

[23]Helen Gaskell, Sheena Derry,R Andrew Moore, and Henry J McQuay.Author informationCopyright and License informationDisclaimerSingle dose oral oxycodone and oxycodone plus paracetamol (acetaminophen) for acute postoperative pain in adults.Cochrane Database Syst Rev. 2009 Jul; 2009(3): CD002763.

[24]Arumugam S, Lau CS, Chamberlain RS.Use of preoperative gabapentin significantly reduces postoperative opioid consumption: a meta-analysis.J Pain Res. 2016 Sep 12;9:631-40. doi:10.2147/JPR.S112626. eCollection 2016.

[25]Stambaugh JE Jr, Drew J.The combination of ibuprofen and oxycodone/acetaminophen in the management of chronic cancer pain.Clin Pharmacol Ther. 1988 Dec;44(6):665-9.

[26]SteinbergAC.Preemptive analgesia for postoperative hysterectomy pain control: systematic review and clinical practice guidelines.Am J Obstet Gynecol. 2017 Sep;217(3):303-313.e6. doi:

10.1016/j.ajog.2017.03.013. Epub 2017 Mar 27.

[27]Axén I.Pain-related Sleep Disturbance: A Prospective Study With Repeated Measures.Clin J Pain. 2016 Mar;32(3):254-9. doi: 10.1097/AJP.0000000000000249.

[28]Bahouq $\mathrm{H}$, Allali F, Rkain $\mathrm{H}$, et al. Prevalence and severity of insomnia in chronic low back pain patients. Rheumatol Int. 2013;33:1277-1281.

[29]Kelly GA, Blake C, Power CK, et al. The association between chronic low back pain and sleep: a systematic review. Clin J Pain. 2011;27:169-181.

[30]Schuh-Hofer S, Wodarski R, Pfau DB, et al. One night of total sleep deprivation promotes a state of generalized hyperalgesia: a surrogate pain model to study the relationship of insomnia and pain. Pain. 2013;154:1613-1621.

[31]Farzi F, Naderi Nabi B, Mirmansouri A, Fakoor F, Atrkar Roshan Z, Biazar G, Zarei T.Postoperative Pain After Abdominal Hysterectomy: A Randomized, Double-Blind, Controlled Trial Comparing the Effects of Tramadol and Gabapentin as Premedication.Anesth Pain Med. 2016 Jan 17;6(1):e32360. doi: 10.5812/aapm.32360. eCollection 2016 Feb. 
[32]ZhangZY,JiaNG,HuangWQ.Safety and efficacy of early oral oxycodone/acetaminophen and tramadol in Chinese gynecology patient undergoing laparoscopy operation[J].Chinese Clinical Pharmacology and Therapeutics,2005(02):168-171.

[33] Grant MC, Betz M, Hulse M, et al. The Effect of Preoperative Pregabalin on Postoperative Nausea and Vomiting: A Meta-analysis. Anesth Analg. 2016;123(5):1100-1107.

[34]Richards P, Gimbel JS, Minkowitz HS, Kelen R, Stern W.Comparison of the efficacy and safety of dualopioid treatment with morphine plus oxycodone versus oxycodone/acetaminophen for moderate to severe acute pain after total knee arthroplasty.Clin Ther. 2013 Apr;35(4):498-511. doi:

10.1016/j.clinthera.2013.03.002. Epub 2013 Mar 29.

[35]Graudins A,Meek R, Parkinson J, Egerton-Warburton D,Meyer A.A randomised controlled trial of paracetamol and ibuprofen with or without codeine or oxycodone as initial analgesia for adults with moderate pain from limb injury.Emerg Med Australas. 2016 Dec;28(6):666-672. doi: 10.1111/17426723.12672. Epub 2016 Sep 7.

[36]Tiippana EMヌHamunen K.囚Kontinen VKखKalso E.Do surgical patients benefit from perioperative gabapentin/pregabalin? A systematic review of efficacy and safety.Anesth Analg. 2007 Jun;104(6):154556 .

[37]Moen V,Dahlgren N, Irestedt L.Severe neurological complicatons after central neuraxial blockades in Sweden 1990-1999.Anesthesiology. 2004;101(4):950-9.

\section{Tables}

Due to technical limitations, the tables have been placed in the Supplementary Files section.

\section{Figures}




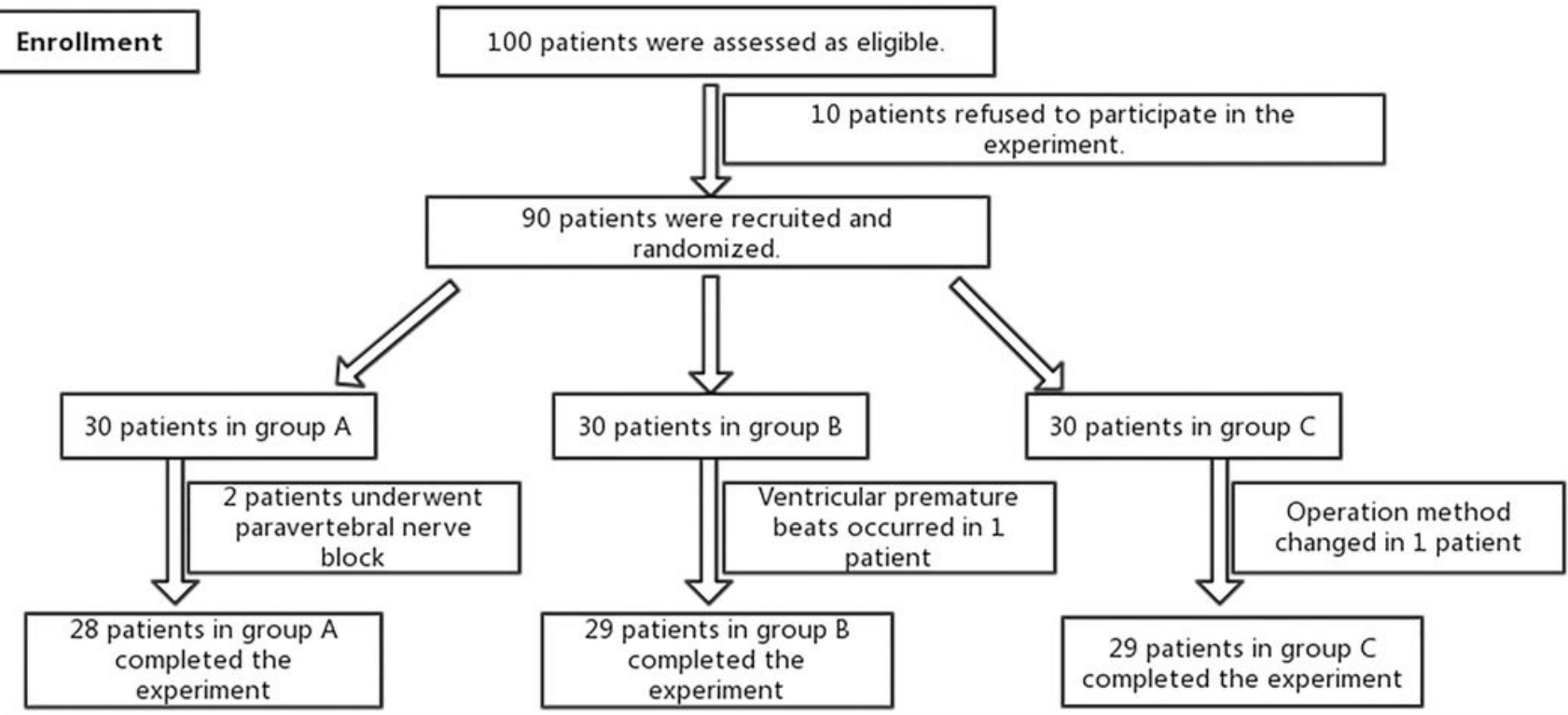

Figure 1.Flow chart of this study. A total of 90 patients were enrolled in this study. Two patient from the group $A$ underwent paravertebral nerve lock.Ventricular premature beats occurredin 1 patients in group B and one patient's operation method changed in group C. Therefore, 86 patients in total had completed the study.

\section{Figure 1}

Figure 2.Comparsion of pain scores at various time points post-operative.
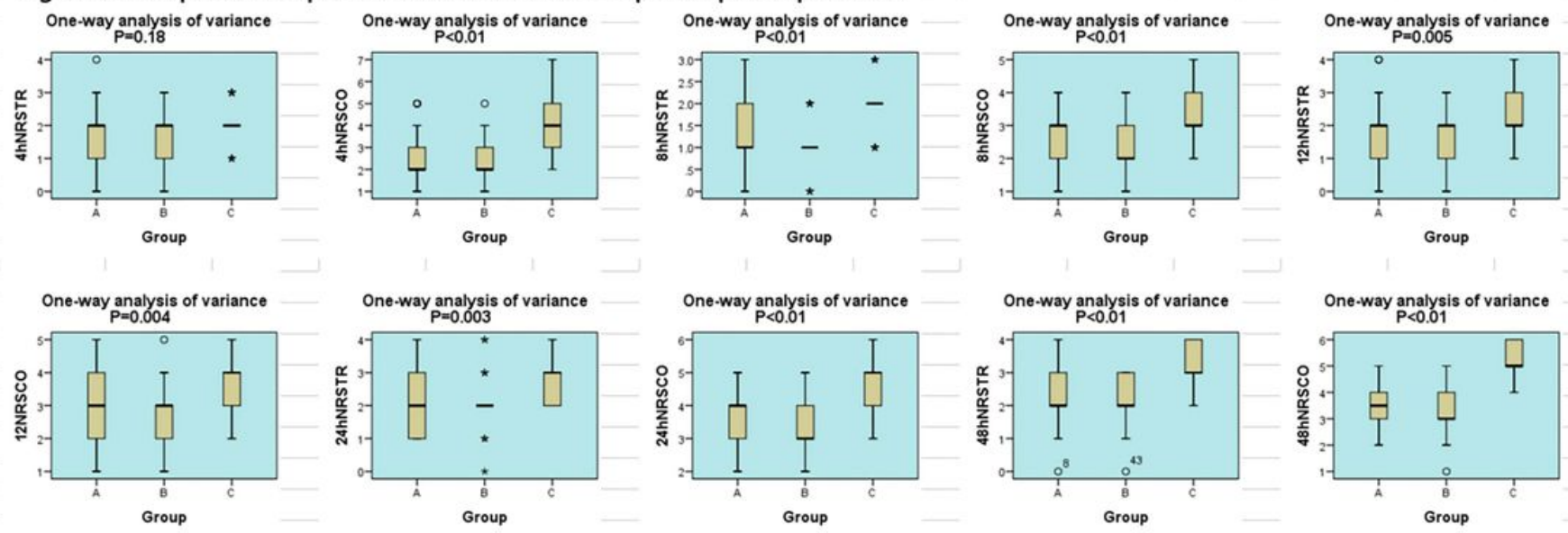

Figure 2 
Figure 3.Comparison of several secondary indicators after surgery
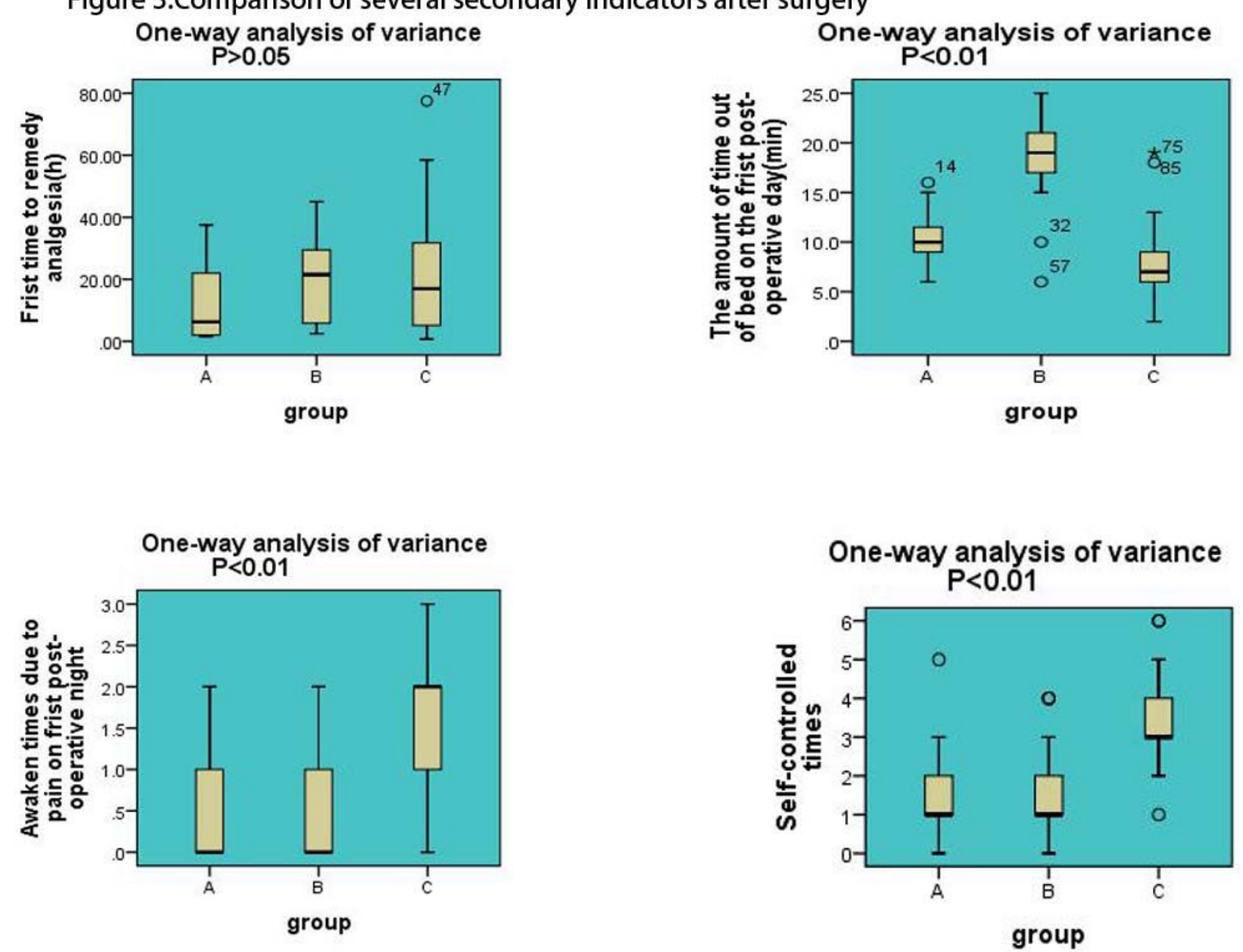

Figure 3

\section{Supplementary Files}

This is a list of supplementary files associated with this preprint. Click to download.

- Table5.tif

- Table8.tif

- Table9.tif

- CONSORT2010Checklist.doc

- Table6.tif

- Table7.tif

- Table2.tif

- Table1.tif 
- Table4.tif

- Table3.tif

Page 17/17 